\title{
Retroperitoneal bile leak after laparoscopic cholecystectomy
}

\author{
Dee Zhen LIM, ${ }^{1}$ Enoch Wong, ${ }^{2}$ Sayed Hassen, ${ }^{2}$ Yahya AL-Habbal ${ }^{2}$
}

${ }^{1}$ School of Medicine, Nursing and Health Science, Monash University, Melbourne, Victoria, Australia

${ }^{2}$ Department of General Surgery, Eastern Health, Box Hill, Victoria, Australia

\section{Correspondence to Yahya AL-Habbal,} yahya@alhabbal.info

Accepted 3 January 2018

\section{SUMMARY}

Bile duct injury (BDI) is a well-recognised complication of laparoscopic cholecystectomy (LC). Following a BDI, bile usually leaks into the peritoneal space and causes biliary peritonitis. This manifests as non-specific abdominal pain and fever occurring several days after the surgery. It can be managed by laparoscopic washout with or without bile duct repair. We present a rare case of retroperitoneal bile leak post-LC. The mechanism of injury here was likely partial avulsion from excessive traction of the cystic duct during intraoperative cholangiogram. Diagnosing retroperitoneal bile leak can be difficult because it is extremely rare and the presenting symptoms can be similar to an intraperitoneal bile leak. A high index of clinical suspicion is required. In cases of suspected bile leak, any mismatch between the exploratory laparoscopic findings and imaging findings should alert surgeons to consider the rare possibility of a retroperitoneal bile leak.

\section{BACKGROUND}

Laparoscopic cholecystectomy (LC) is the gold standard surgical management for a spectrum of cholelithiasis-related disorders. It is one of the most commonly performed surgical procedures worldwide. One of the known complications of LC includes bile duct injuries (BDIs). In recent studies, LC has a BDI rate of approximately $0.1 \%-0.6 \% .^{1-3}$

Following BDI, bile can leak into the peritoneal cavity - a potential space between the visceral and parietal peritoneum. ${ }^{4}$ Intraperitoneal bile leak can cause a localised bile collection (biloma), abscess formation or peritonitis. ${ }^{5}$ Patients usually present between day 3 and 6 after LC with symptoms such as non-specific abdominal pain, fever, anorexia, malaise, jaundice, nausea and vomiting. ${ }^{56}$ Liver function test may show hyperbilirubinaemia and a cholestatic pattern. ${ }^{56}$ If the diagnosis of an intraperitoneal bile leak is delayed or missed, patients can

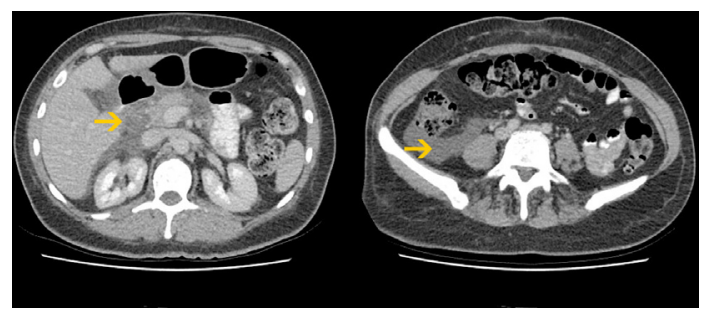

To cite: LIM DZ, Wong E, Hassen $\mathrm{S}$, et al. BMJ Case Rep Published Online First: [please include Day Month Year]. doi:10.1136/bcr-2017222750

Check for updates
Figure 1 The first CT scan showing free fluid around the gallbladder fossa, pancreas, perihepatic space, Morrison's pouch, paracolic gutter and pelvis.

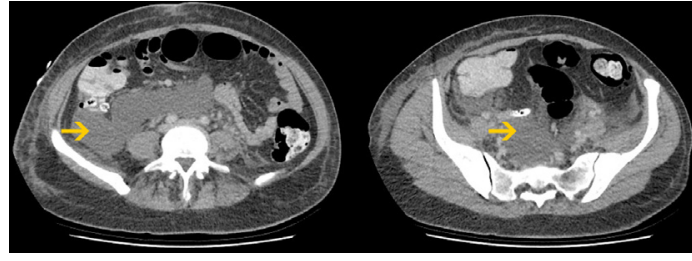

Figure 2 The second CT scan showing fluid in the retroperitoneal space, right paracolic gutter, right iliac fossa and pelvis.

develop serious complications such as sepsis, recurrent cholangitis and secondary biliary cirrhosis, resulting in significant morbidity and mortality. ${ }^{6}$

It is extremely rare for LC to cause bile leakage into the retroperitoneal space instead of the peritoneal cavity. The retroperitoneal space is a space bounded by the posterior parietal peritoneum and the transversalis fascia. ${ }^{4}$ To our knowledge, there are only three documented cases of retroperitoneal bile leak post-LC in the current literature. ${ }^{78}$

Leakage of bile into the retroperitoneal space can occur via several mechanisms. The distal biliary system including the duodenum, where bile eventually drains into, is all retroperitoneal structures. Injuries at any of these locations can predispose to a retroperitoneal bile leak. Such injuries can be either spontaneous or iatrogenic. Spontaneous
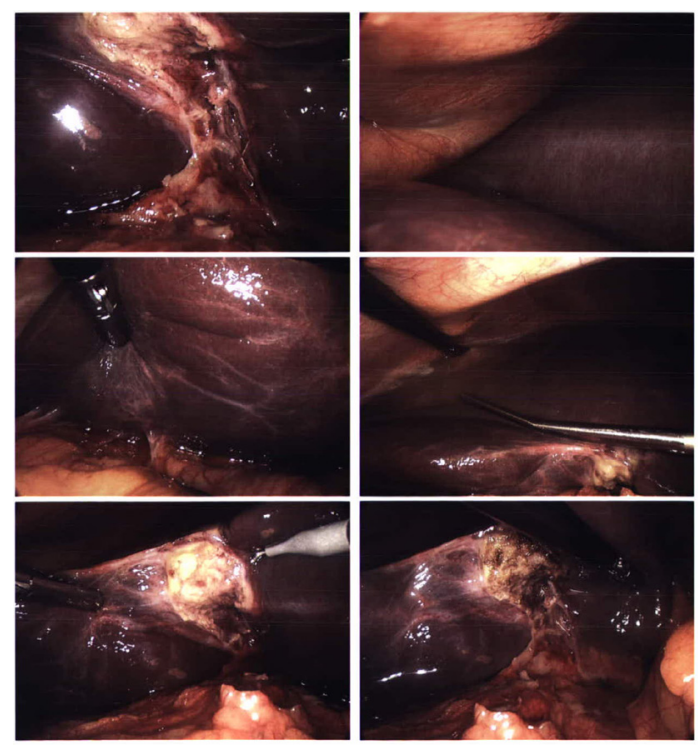

Figure 3 Intraoperative photography from the second laparoscopic washout showing minimal bile visualised within the intraperitoneal cavity. 


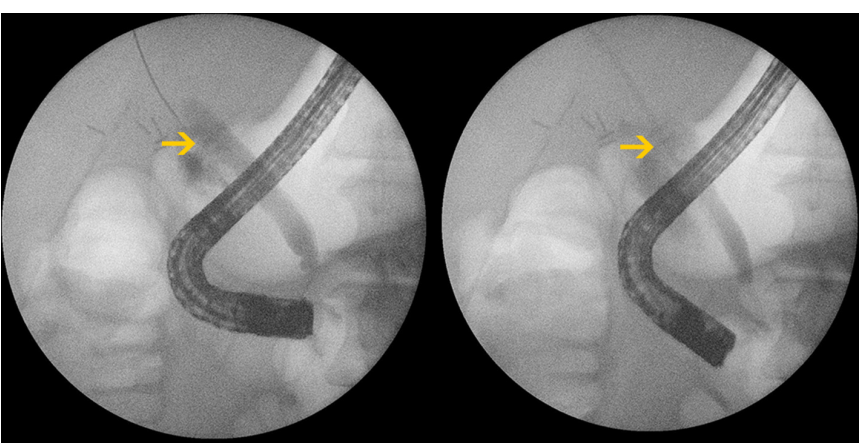

Figure 4 ERCP showing extravasation of contrast from the cysticocholedochal junction. ERCP, endoscopic retrograde cholangiopancreatography.

hepatic duct and common bile duct (CBD) perforation have been documented to cause retroperitoneal bile leak in several case reports. $^{9-11}$ For iatrogenic injuries, duodenal or periampullary perforation post-endoscopic retrograde cholangiopancreatography (ERCP) can cause retroperitoneal bile leak. ${ }^{12-14} \mathrm{LC}$ is not known to cause retroperitoneal bile leak because any erroneous surgical dissection will cut the investing peritoneum and causes bile to leak mainly into the peritoneal cavity. Although retroperitoneal bile leak after LC is an extremely rare complication, it is very important for surgeons to be aware of it. Failure to consider the possibility of a retroperitoneal bile leak can result in delayed diagnosis, ineffective management and poor patient outcomes. In this case report, we detail our experience with diagnosing and managing a case of retroperitoneal bile leak post-LC.

\section{CASE PRESENTATION}

A 44-year-old woman presented to the emergency department with acute epigastric pain, vomiting and chills 4 days post-LC for cholelithiasis. No significant operative difficulties were reported for the LC. The intraoperative cholangiogram (IOC) was normal, and no drain tube was required. On presentation, she was haemodynamically stable, afebrile and not jaundiced. Her right upper quadrant was tender to palpation but soft. Her only previous abdominal surgery was a laparoscopic tubal ligation 5 years prior.

\section{INVESTIGATIONS}

Initial blood tests showed neutrophilia of $19.7 \times 10^{9} / \mathrm{L}$ (reference: $2.0-7.0 \times 10^{9} / \mathrm{L}$ ) and a $\mathrm{C}$ reactive protein of $11 \mathrm{mg} / \mathrm{L}$ (reference:

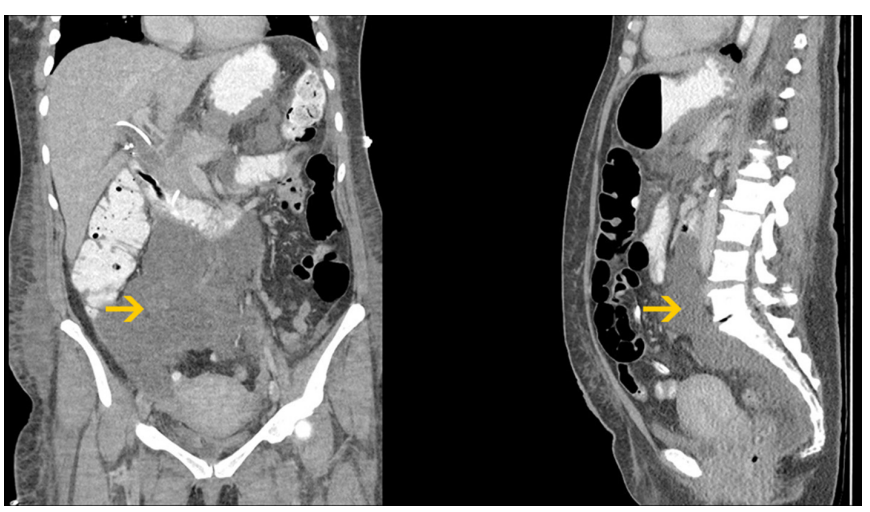

Figure 5 The third CT scan showing a large retroperitoneal collection extending from the posterior aspect of the third part of duodenum to the right paracolic gutter.
$<5 \mathrm{mg} / \mathrm{L})$. Liver function test was normal. Total bilirubin was 3 $\mu \mathrm{mol} / \mathrm{L}$ (reference: $<22 \mu \mathrm{mol} / \mathrm{L}$ ), and serum lipase was $17 \mathrm{IU} / \mathrm{L}$ (reference: $<60 \mathrm{IU} / \mathrm{L}$ ). The first CT abdomen and pelvis with contrast showed free fluid around the gallbladder fossa, liver, pancreas, paracolic gutter and pelvis (figure 1). Cholescintigraphy showed marked tracer accumulation in the gallbladder fossa. The initial impression was that the patient had biliary peritonitis from an intraperitoneal bile leak. Consequently, the patient underwent a laparoscopic washout. There was minimal bile in the peritoneal cavity, but given the high index of suspicion, a subhepatic drain was still placed. The patient did not improve after the washout. Drain output was minimal, and blood tests showed rising leucocytosis and inflammatory markers. Given the failure to improve, a second CT abdomen and pelvis was performed, which showed a fluid collection in the retroperitoneal space, right paracolic gutter, right iliac fossa and pelvis (figure 2). The impression was that the patient had an accumulating intraperitoneal bile leak due to an ineffective subhepatic drainage. The retroperitoneal fluid was thought to be reactionary to the bile leak. Consequently, a second laparoscopic washout was performed, and two wider bore drain tubes were inserted (to right upper quadrant and pelvis). In the second washout, there was also only minimal amount of bile in the peritoneal space (figure 3). After the second washout, the patient did not improve, and drain outputs remained low despite interval increase in the size of collections on CT scan. We noted that both laparoscopic findings were inconsistent with the imaging findings. On day 10 of admission, ERCP was performed, which showed a high-grade bile leak from the choledochocystic junction (figure 4). Post-ERCP CT abdomen and pelvis demonstrated a large retroperitoneal collection extending from the posterior aspect of the third part of the duodenum to the right paracolic gutter, measuring $16.8 \mathrm{~cm}$ in the maximal axial dimensions (figure 5). The patient was diagnosed with a retroperitoneal bile leak post-LC.

\section{DIFFERENTIAL DIAGNOSIS}

- Intraperitoneal bile leak.

- Retroperitoneal bile leak.

- Duodenal perforation.

\section{TREATMENT}

The retroperitoneal collection was drained percutaneously using a 10 French Dawson Mueller drain tube inserted under CT guidance. Bilious fluid was aspirated, and fluid lipase was measured at only $15 \mathrm{IU} / \mathrm{L}$. The biliary leak was treated endoscopically with sphincterotomy and a 7 French $5 \mathrm{~cm}$ plastic double pigtail stent. The leak persisted despite the plastic stent. It resolved after the plastic stent was exchanged for an $8 \mathrm{~cm} \times 10 \mathrm{~mm}$ fully covered metal stent. The patient also received empirical antibiotics cover during her hospital stay. She improved clinically following metal stent insertion and was discharged on day 20.

\section{OUTCOME AND FOLLOW-UP}

The retroperitoneal drain and the covered stent were removed after 3 weeks and 6 weeks, respectively. The patient had a full recovery.

\section{DISCUSSION}

Retroperitoneal bile leak is an extremely rare complication of LC-related BDI. We searched the PubMed database for similar cases using the keyword: retroperitone* and the medical subject heading term: cholecystectomy. We then performed a citation 


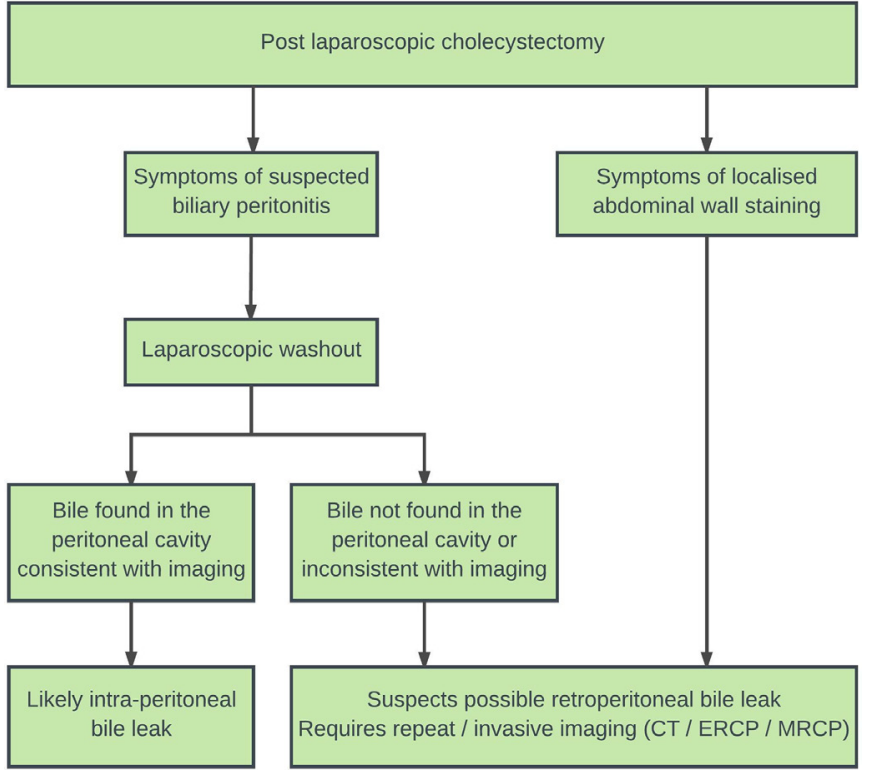

Figure 6 Proposed algorithm for identifying a retroperitoneal bile leak. ERCP, endoscopic retrograde cholangiopancreatography.

search on all relevant English-language studies with the Web of Science database. To our knowledge, there are only three reported cases of retroperitoneal bile leak after LC in the current literature. ${ }^{78} \mathrm{McAlister}$ and Sener reported two cases of this unusual complication. ${ }^{7}$ Both of their case patients presented 4 to 5 days post-LC with yellow abdominal wall staining. ${ }^{7}$ One patient had yellow staining of the bilateral flanks and groins, whereas the other patient had a more extensive staining from the upper torso to the thigh and perineum. ${ }^{7}$ In the latter case, the yellow stain was clearly delineated at $3 \mathrm{~cm}$ below the clavicle and $3 \mathrm{~cm}$ below the groin skin, where Scarpa's fascia merges with the clavipectoral fascia and the fascia lata of the thigh - a sign called icterus marginatus. ${ }^{7}$ More recently, Shahedi and Tejaswi also reported a case with similar clinical presentation. ${ }^{8}$ In all these reported cases, the retroperitoneal bile leak was relatively benign. None required surgical interventions, and all resolved with endoscopic interventions such as biliary stent or sphincterotomy with or without percutaneous drainage. Unlike these cases, our case patient presented with biliary peritonitis from a retroperitoneal bile leak. The clinical course was significantly more complicated involving multiple interventions including as two exploratory laparoscopies, two biliary stents and a retroperitoneal drain placement.

The precise aetiology of a retroperitoneal bile leak after LC is unclear. In the three reported cases, the origin of the bile leak was cited as the cystic stump. ${ }^{78}$ Our case patient had a partial tear at the junction of the cystic duct and CBD distal from the surgical clips, as seen on the ERCP. We believe that the mechanism of injury was likely due to partial avulsion from excessive traction of the cystic duct during IOC. Another less likely but plausible explanation is a traumatic injury during the insertion of the IOC catheter. The junction of cystic duct and CBD is covered by peritoneum and partially by the lesser omentum. Typical BDI occurs because of erroneous dissection which damages both the bile duct and its investing peritoneum. This causes bile to leak mainly into the peritoneal cavity. We speculate that an avulsion injury is unique because it preserves the investing peritoneum. As such, the bile leak is contained within the hepatoduodenal ligament. The bile can then track downwards until where the peritoneum diverges and escapes into the retroperitoneal space. Such a mechanism has been described before in a case of retroperitoneal bile leak that was not due to cholecystectomy. ${ }^{15}$ In 1990, Cólović and Perisić-Savić performed a surgical exploration on a patient who had right lower quadrant abdominal pain after a severe vomiting episode on the background of recurrent cholangitis from a Bismuth 3 stricture after cholecystectomy. ${ }^{15}$ They found that the patient had an internal biliary fistula that extended along the hepatoduodenal ligament to behind the duodenum and into the retroperitoneal space. ${ }^{15}$

There are many classification systems for BDI. In the prelaparoscopic era, the Bismuth classification was commonly used. The Bismuth classification is based on the level of injury in the CBD, from distal to proximal. ${ }^{16}$ With LC, BDI is becoming more proximal and complex in nature. ${ }^{2}$ More comprehensive classification systems, such as the Strasberg classification that includes non-CBD injuries or the Stewart-Way and Hannover classifications that include vascular injuries, have been described. ${ }^{17}{ }^{18}$ The BDI in our case fits most closely with a type II injury described in the Csendes classification. ${ }^{19}$ The Csendes type II injury refers to lesions that occur at the cysticocholedochal junction from various mechanisms including excessive traction, use of a Dormia catheter, section of the cystic duct close to the CBD and electrocautery injuries. ${ }^{19}$ However, we believe that retroperitoneal bile leak represents a unique and separate entity. It occurs through a distinctive mechanism and poses unique diagnostic and management challenges that the current classification system failed to address.

In our experience, the main challenge of a retroperitoneal bile leak is the diagnostic process. Unlike other cases in the literature, our case patient presented with symptoms that closely mimic an intraperitoneal bile leak. The initial imagings were also unable to distinguish between an intraperitoneal versus a retroperitoneal bile leak clearly. The first CT abdomen and pelvis could not localise the bile collection accurately, probably because the bile volume was low. As a result, the patient was managed initially as an intraperitoneal bile leak and received multiple laparoscopic washouts. The second CT abdomen and pelvis did demonstrate some retroperitoneal collection. However, retroperitoneal bile leak is not a known complication of LC. As such, we had no clinical suspicion, and the significance of this finding was not immediately recognised. We started to consider other differential diagnosis when we observed a consistent discrepancy between the laparoscopic findings and imaging findings. On the CT, there was a sizeable bile collection; however, on laparoscopic examination, there was only minimal bile collection in the peritoneal space. The diagnosis of retroperitoneal bile leak was made after we performed invasive imaging with ERCP and repeated another CT abdomen and pelvis. It took 11 days before a retroperitoneal drain was inserted. Based on our experience, we like to propose an algorithm to help other surgical teams identify any future retroperitoneal bile leak post-LC (figure 6).

The management principle in retroperitoneal bile leak is similar to that of intraperitoneal bile leak. The management goals are first to drain the collection and second to stop the ongoing bile leak. To drain a retroperitoneal collection, percutaneous ultrasound or CT-guided drainage can be used. To stop the ongoing bile leak, there are various therapeutic options available. The optimal intervention will depend on many factors including the location, mechanism and severity of the injury. In mild BDI, drainage of the bile collection alone may be sufficient. In moderate BDI with no loss of bile duct continuity, endoscopic interventions such as sphincterotomy and/or biliary stent can be used. $^{20} 21$ These interventions decrease the transpapillary 


\section{Learning points}

- Retroperitoneal bile leak is a rare complication of laparoscopic cholecystectomy.

- While retroperitoneal bile leak may present as a distinctive localised abdominal wall staining, it can also present with symptoms similar to an intraperitoneal bile leak.

- Any mismatch between the exploratory laparoscopic findings and imaging findings should alert the surgeons to consider the rare possibility of a retroperitoneal bile leak.

- Absence of intraperitoneal bile on laparoscopy does not rule out ongoing bile leak.

- Excessive traction of the cystic duct during intraoperative cholangiogram can cause an avulsion injury and should be avoided.

pressure gradient and promote preferential bile flow through the main duct instead of the leak site, giving the leak site time to heal. ${ }^{2021}$ Metal biliary stents may confer the additional benefits of physically bridging the mucosal gap due to their larger diameter. ${ }^{22}$ Fully covered metal stents have been used to manage refractory biliary leak cases with great success. ${ }^{22-24}$ Surgical repair such as hepaticojejunostomy is indicated when there is a loss of bile duct continuity.

Prevention of BDI is an important aspect of LC. Specific techniques such as the critical view of safety may help to prevent BDI by reducing the chances of misidentifying structures and erroneous dissection. ${ }^{25}$ However, these techniques do not help to prevent the BDI seen in our case patient. To prevent the avulsion injury seen in our case, we recommend that surgeons should avoid excessive traction on the cystic duct during IOC.

Contributors DZL performed the literature review and drafted the manuscript. EW, YA-H and SH were responsible for providing guidance, critical revision, editing, proofreading and supervision for the manuscript.

\section{Competing interests None declared.}

Patient consent Obtained.

Provenance and peer review Not commissioned; externally peer reviewed.

(c) BMJ Publishing Group Ltd (unless otherwise stated in the text of the article) 2018. All rights reserved. No commercial use is permitted unless otherwise expressly granted.

\section{REFERENCES}

1 Viste A, Horn A, Øvrebø K, et al. Bile duct injuries following laparoscopic cholecystectomy. Scand J Surg 2015:104:233-7.
2 Chuang KI, Corley D, Postlethwaite DA, et al. Does increased experience with laparoscopic cholecystectomy yield more complex bile duct injuries? Am J Surg 2012;203:480-7

3 Mahatharadol V. Bile duct injuries during laparoscopic cholecystectomy: an audit of 1522 cases. Hepatogastroenterology 2004;51:12-14.

4 Tirkes T, Sandrasegaran K, Patel AA, et al. Peritoneal and retroperitoneal anatomy and its relevance for cross-sectional imaging. Radiographics 2012;32:437-51.

5 Massoumi H, Kiyici N, Hertan H. Bile leak after laparoscopic cholecystectomy. J Clin Gastroenterol 2007:41:301-5.

6 Conzo G, Napolitano S, Candela G, et al. latrogenic Bile Duct Injuries Following Laparoscopic Cholecystectomy: Myth or Reality? A Recent Literature Review from 2006 to 2011. Tripodi V, editor: InTech 2012.

7 McAlister VC, Sener A. Demarcated truncal jaundice: a sign of retroperitoneal bile leakage. Ann Intern Med 2005;142:389.

8 Shahedi K, Tejaswi S. Abdominal Wall Bile Staining Without Jaundice. Clin Gastroenterol Hepatol 2016;14:A33-A34.

9 Brady RR, McAteer E, Weir CD. Biliscrotum and retroperitoneal biloma: spontaneous rupture of the biliary system presenting as an incarcerated inguinal hernia. Ulster Med J 2006:75:85-7.

10 Yaşar NF, Yaşar B, Kebapçı M. Spontaneous common bile duct perforation due to chronic pancreatitis, presenting as a huge cystic retroperitoneal mass: a case report. Cases J 2009;2:6273.

11 Ishii K, Matsuo K, Seki H, et al. Retroperitoneal Biloma due to Spontaneous Perforation of the Left Hepatic Duct. Am J Case Rep 2016:17:264-7.

12 Allegue F, Pérez-Pérez L, Maza MT, et al. Localized abdominal wall bile staining due to retroperitoneal bile leak. Acta Derm Venereol 2009;89:410-1.

13 Machado NO. Management of duodenal perforation post-endoscopic retrograde cholangiopancreatography. When and whom to operate and what factors determine the outcome? A review article. JOP 2012;13:18-25.

14 Wu HM, Dixon E, May GR, et al. Management of perforation after endoscopic retrograde cholangiopancreatography (ERCP): a population-based review. HPB 2006:8:393-9.

15 Cólović R, Perisić-Savić M. Retroperitoneal biloma secondary to operative common bile duct injury. HPB Surg 1991;3:193-7.

16 Bismuth H, Majno PE. Biliary strictures: classification based on the principles of surgical treatment. World J Surg 2001;25:1241-4.

17 Mercado MA, Domínguez I. Classification and management of bile duct injuries. World J Gastrointest Surg 2011;3:43-8.

18 Chun K. Recent classifications of the common bile duct injury. Korean J Hepatobiliary Pancreat Surg 2014;18:69-72.

19 Csendes A, Navarrete C, Burdiles P, et al. Treatment of common bile duct injuries during laparoscopic cholecystectomy: endoscopic and surgical management. World J Surg 2001;25:1346-51.

20 Kim KH, Kim TN. Endoscopic management of bile leakage after cholecystectomy: a single-center experience for 12 years. Clin Endosc 2014;47:248-53.

21 Nawaz H, Papachristou GI. Endoscopic treatment for post-cholecystectomy bile leaks: update and recent advances. Ann Gastroenterol 2011;24:161-3.

22 Mangiavillano B, Pagano N, Baron TH, et al. Outcome of stenting in biliary and pancreatic benign and malignant diseases: A comprehensive review. World J Gastroenterol 2015;21:9038-54.

23 Canena J, Horta D, Coimbra J, et al. Outcomes of endoscopic management of primary and refractory postcholecystectomy biliary leaks in a multicentre review of 178 patients. BMC Gastroenterol 2015;15:105

24 Lalezari D, Singh I, Reicher $S$, et al. Evaluation of fully covered self-expanding metal stents in benign biliary strictures and bile leaks. World J Gastrointest Endosc 2013;5:332-9

25 Eikermann M, Siegel R, Broeders I, et al. Prevention and treatment of bile duct injuries during laparoscopic cholecystectomy: the clinical practice guidelines of the European Association for Endoscopic Surgery (EAES). Surg Endosc 2012;26:3003-39.

Copyright 2017 BMJ Publishing Group. All rights reserved. For permission to reuse any of this content visit

http://group.bmj.com/group/rights-licensing/permissions.

BMJ Case Report Fellows may re-use this article for personal use and teaching without any further permission.

Become a Fellow of BMJ Case Reports today and you can:

- Submit as many cases as you like

- Enjoy fast sympathetic peer review and rapid publication of accepted articles

- Access all the published articles

- Re-use any of the published material for personal use and teaching without further permission

For information on Institutional Fellowships contact consortiasales@bmjgroup.com

Visit casereports.bmj.com for more articles like this and to become a Fellow 